\title{
Direct observation of Oersted-field-induced magnetization dynamics in magnetic nanostripes
}

\author{
V. Uhlî́,,${ }^{1,2}$ S. Pizzini, ${ }^{1}$ N. Rougemaille, ${ }^{1}$ V. Cros,${ }^{3}$ E. Jiménez, ${ }^{4}$ L. Ranno, ${ }^{1}$ O. Fruchart, ${ }^{1}$ M. Urbánek,${ }^{2}$ G. Gaudin, ${ }^{5}$ \\ J. Camarero, ${ }^{4}$ C. Tieg, ${ }^{6}$ F. Sirotti, ${ }^{7}$ E. Wagner, ${ }^{1}$ and J. Vogel ${ }^{1}$ \\ ${ }^{1}$ Institut Néel, Centre national de la recherche scientifique and Université Joseph Fourier, BP166, F-38042 Grenoble, France \\ ${ }^{2}$ Institute of Physical Engineering, Brno University of Technology, CZ-61669 Brno, Czech Republic \\ ${ }^{3}$ Unité Mixte de Physique CNRS-Thales, Route départementale 128, F-91767 Palaiseau Cedex, France \\ ${ }^{4}$ Departamento Física de la Materia Condensada, Instituto "Nicolás Cabrera" and Instituto Madrileño de Estudios Avanzados-Nanociencia, \\ Campus Universidad Autónoma de Madrid, E-28049 Madrid, Spain \\ ${ }^{5}$ SPINTEC, UMR8191 CEA/CNRS/UJF/Grenoble INP, INAC, F-38045 Grenoble, France \\ ${ }^{6}$ European Synchrotron Radiation Facility, BP200, F-38043 Grenoble, France \\ ${ }^{7}$ Synchrotron SOLEIL, L'Orme des Merisiers, Saint-Aubin, F-91192 Gif-sur-Yvette, France
}

(Received 26 November 2010; published 12 January 2011)

\begin{abstract}
We have used time-resolved $\mathrm{x}$-ray photoemission electron microscopy to investigate the magnetization dynamics induced by nanosecond current pulses in $\mathrm{NiFe} / \mathrm{Cu} / \mathrm{Co}$ nanostripes. A large tilt of the $\mathrm{NiFe}$ magnetization in the direction transverse to the stripe is observed during the pulses. We show that this effect cannot be quantitatively understood from the amplitude of the Oersted field and the shape anisotropy. High-frequency oscillations observed at the onset of the pulses are attributed to precessional motion of the NiFe magnetization about the effective field. We discuss the possible origins of the large magnetization tilt and the potential implications of the static and dynamic effects of the Oersted field on current-induced domain-wall motion in such stripes.
\end{abstract}

DOI: 10.1103/PhysRevB.83.020406

PACS number(s): 75.70.Ak, 07.85.Qe, 75.50.Bb, 75.60.Jk

The possibility of manipulating the magnetic configuration of nanostructures by using electrical currents is a recent, exciting development in spintronics. Electrical currents can affect the magnetization of magnetic nanostructures through both the charge and the spin of the conduction electrons. In recent years it has been shown that spin-transfer torque $(\mathrm{STT})^{1,2}$ and Rashba spin-orbit torque effects ${ }^{3}$ act on the magnetization, in addition to the classical Oersted magnetic field $H_{\mathrm{Oe}}$. In general, the combination of these effects should be taken into account in the description of the magnetization dynamics during the application of a current pulse. For instance, it was shown that the contribution of the Oersted field and not only STT is needed to explain the magnetization reversal in trilayered pillars induced by a current flowing perpendicular to the plane of the layers. ${ }^{4,5}$ For in-plane currents, $H_{\mathrm{Oe}}$ has been invoked to explain magnetization reversal in mesoscopic $\mathrm{NiFe} / \mathrm{Cu} / \mathrm{Co} / \mathrm{Au}_{\text {bars }}{ }^{6}$ and the resonant depinning of constricted domain walls (DWs) in $\mathrm{NiFe} / \mathrm{Cu} / \mathrm{Co}$ trilayers. $^{7}$

Several studies on the effects of current pulses on the magnetization of nanostripes, mainly concerning currentinduced domain-wall motion (CIDM), have been based on the observation of the domain structure before and after the application of a current pulse. ${ }^{8,9}$ However, the effect of the Oersted field on the magnetization can only be investigated by direct, dynamic observations during the current pulses. This has been achieved in this work, using time-resolved $\mathrm{x}$-ray magnetic circular dichroism combined with photoemission electron microscopy (XMCD-PEEM). Our results show that the current-induced field during nanosecond pulses causes both quasistatic and precessional effects on the $\mathrm{NiFe}$ magnetization. These effects may contribute to the increased efficiency of current-induced domain-wall motion observed in such trilayers. ${ }^{10-12}$
Stacks of $\mathrm{Cu}(2 \mathrm{~nm}) / \mathrm{Ni}_{80} \mathrm{Fe}_{20}(5 \mathrm{~nm}) / \mathrm{Cu}(5 \mathrm{~nm}) / \mathrm{Co}(5 \mathrm{~nm}) /$ $\mathrm{CoO}(6 \mathrm{~nm})$ deposited on highly resistive $\mathrm{Si}(100)(\rho>$ $300 \Omega \mathrm{cm}$ ) were patterned in 400-nm-wide zigzag stripes, with angles of $90^{\circ}$ and $13-\mu \mathrm{m}$-long straight sections, combining electron-beam lithography and ion-beam etching. Contact electrodes made of $\mathrm{Ti} / \mathrm{Au}$ were subsequently deposited using evaporation and a lift-off technique. Prior to the XMCD-PEEM measurements, most of the $2-\mathrm{nm} \mathrm{Cu}$ protective layer was removed in situ using Ar bombardment to increase the XMCD signal of the NiFe layer.

XMCD-PEEM measurements were performed at the synchrotron SOLEIL (TEMPO beamline), using a Focus IS-PEEM. The magnetic configuration in the NiFe layer was imaged by measuring the Ni XMCD intensity, tuning the X-ray energy to the $\mathrm{Ni} L_{3}$ absorption edge $(852.8 \mathrm{eV})$. To optimize the magnetic contrast, the difference between two consecutive images obtained with $100 \%$ left and right circularly polarized $\mathrm{x}$ rays was computed. For each circular polarization, 60 images of $0.5 \mathrm{~s}$ were summed, after correcting for possible image drifts.

Temporal resolution was obtained by synchronizing nanosecond current pulses applied to the nanostripes with the SOLEIL eight-bunch mode, where photon bunches arrive at the sample with a repetition rate of $6.77 \mathrm{MHz}$. The temporal evolution of the magnetic configuration in the nanostripes was obtained by recording images for different delays between the current and photon pulses. ${ }^{13-16}$ If events are reproducible and reversible for each current pulse, the temporal resolution of this pump-probe technique is limited only by the duration of the photon pulses (50-60 ps). The total acquisition time of $1 \mathrm{~min}$ for each XMCD image implies that sequences of about $4 \times 10^{8}$ current (pump) and photon (probe) pulses were averaged. In order to avoid electrical discharges, the voltage between the sample and the objective lens of the PEEM was set to $5.4 \mathrm{keV}$ 


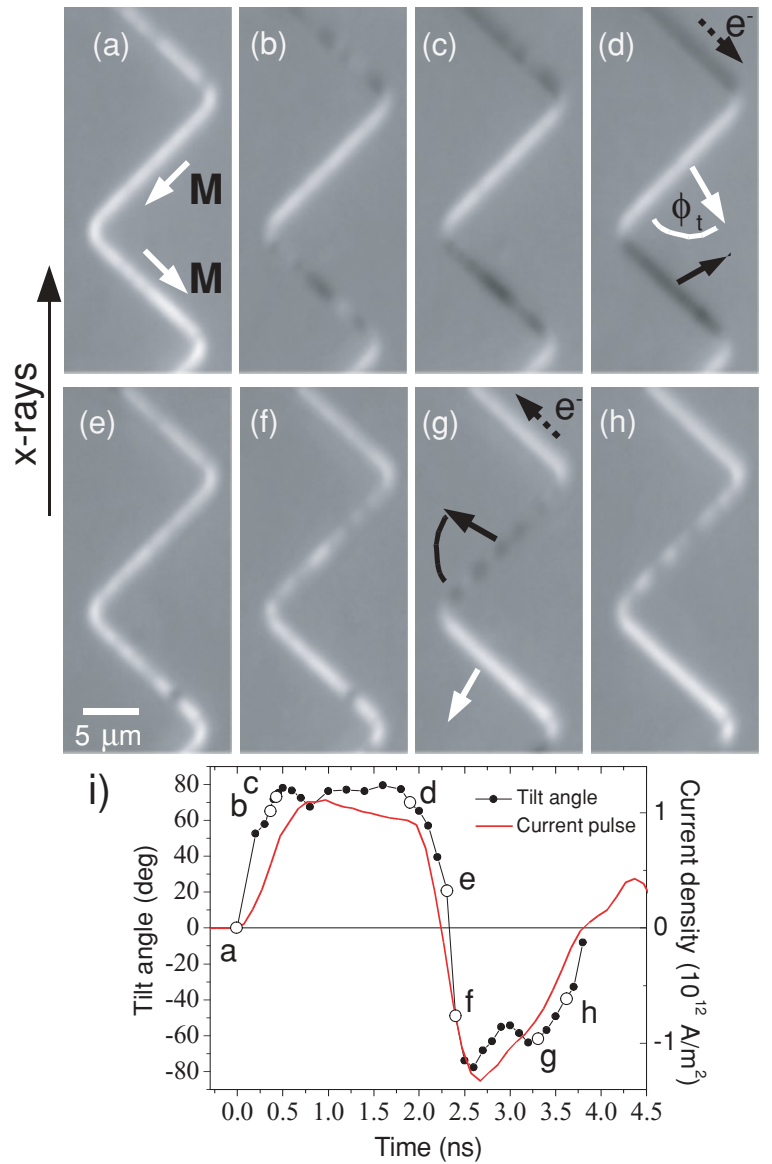

FIG. 1. (Color online) Time-resolved XMCD-PEEM images of the $\mathrm{NiFe}$ layer of a 400 -nm-wide nanostripe at time delays of (a) $0 \mathrm{~ns}$, (b) $0.35 \mathrm{~ns}$, (c) $0.45 \mathrm{~ns}$, (d) $1.9 \mathrm{~ns}$, (e) $2.3 \mathrm{~ns}$, (f) $2.4 \mathrm{~ns}$, (g) 3.3, and (h) $3.6 \mathrm{~ns}$ with respect to the beginning of the positive part of the bipolar current pulse. ${ }^{17}$ (i) These delays are indicated on the bipolar pulse, together with the magnetization tilt angle $\varphi_{t}$. The oscillations in $\varphi_{t}$ at the beginning of the positive and negative parts of the pulse indicate magnetization precession about $H_{\mathrm{Oe}}$.

instead of the nominal $12 \mathrm{keV}$, limiting the spatial resolution to about $0.6 \mu \mathrm{m}$.

Figure 1 shows a series of XMCD-PEEM images of the $\mathrm{NiFe}$ magnetization acquired during the application of bipolar current pulses. The positive (negative) part of the pulse is about 2 ns (1 ns) long, with a maximum amplitude of $+7 \mathrm{~mA}(-9 \mathrm{~mA})$. The latter value corresponds to a current density of $1.5 \times 10^{12} \mathrm{~A} / \mathrm{m}^{2}$, assuming a homogeneous current distribution in the stack. Before and after the current pulses, the magnetization is aligned along the stripe axis, and no domain walls are present, leading to an almost-homogeneous XMCD intensity [Fig. 1(a)]. During the current pulses, the $\mathrm{NiFe}$ magnetization tilts away from the stripe axis, with a tilt angle $\varphi_{t}$. This tilt is counterclockwise for a positive and clockwise for a negative current direction, as can be inferred from the magnetic contrast in the differently oriented sections of the nanostripe [Figs. 1(d) and 1(g)]. The approximate magnetization directions in two of the stripe sections are indicated before the current pulses [Fig. 1(a)] and at the end of the plateau of the positive [Fig. 1(d)] and negative part of the pulses [Fig. 1(g)]. In Figs. 1(d) and 1(g) the electron flow directions are also indicated. The delays between the beginning of the current pulse and the photon pulses at which the images were acquired are shown in Fig. 1(i). ${ }^{17}$

In order to obtain the tilt angle $\varphi_{t}$ as a function of time during the current pulses [Fig. 1(i)], the normalized $\mathrm{XMCD}$ intensity in the bends of the stripe was determined from the XMCD-PEEM images. The XMCD intensity is proportional to the cosine of the angle between the incoming $\mathrm{x}$ rays and the local magnetization; thus, for the bends, $I_{\mathrm{XMCD}} \propto M \cos \left(\varphi_{t}\right) \cos (\alpha)$, where $M$ is the magnetization and $\alpha$ is the angle between the x-ray incidence direction and the sample surface. This angle is constant $\left(25^{\circ}\right)$, and we also suppose the magnetization vector has a constant amplitude. No change in the magnetic contrast due to current-induced heating was observed. At zero current, the magnetization is parallel to the stripe axis and thus $\varphi_{t}=0^{\circ}$, giving $I_{\mathrm{XMCD}}=$ $M \cos (\alpha)=I_{0}$. Then $\varphi_{t}$ can be determined from the different images by $\varphi_{t}=\arccos \left(I_{\mathrm{XMCD}} / I_{0}\right)$.

The NiFe magnetization tilt induced by the transverse Oersted field is surprisingly large, with a value of about $75^{\circ}$ at the end of the positive part of the pulse. For a soft magnetic material such as $\mathrm{NiFe}$, the magnetization direction in a nanostripe is mainly determined by magnetostatic effects, which favor magnetization along the stripe axis. For a 5-nmthick, 400-nm-wide stripe the transverse demagnetizing factor is about $0.023 .^{18} \mathrm{In}$ a first approximation, this would mean that a transverse field of $0.023 \times \mu_{0} M_{S} \times \sin 75^{\circ}=22 \mathrm{mT}$ (with $\mu_{0} M_{S}=1 \mathrm{~T}$ for permalloy) would be required to obtain $\varphi_{t}=75^{\circ}$.

The Oersted field inside a stripe with a rectangular cross section is given by $B_{x}=\mu_{0} J z$, where $J$ is the current density and $z$ is the distance from the stripe axis. A current of $+7 \mathrm{~mA}$ corresponds to a current density of $1.17 \times 10^{12} \mathrm{~A} / \mathrm{m}^{2}$, yielding an average field acting on the NiFe magnetization of $H_{\mathrm{Oe}}=7.4$ $\mathrm{mT}$ if we assume a homogeneous current distribution over the $\mathrm{NiFe} / \mathrm{Cu} / \mathrm{Co}$ trilayer structure and $11 \mathrm{mT}$ for a current flowing entirely through the $\mathrm{Cu}$ and Co layers. With a field of $11 \mathrm{mT}$, the expression given above yields a $\varphi_{t}$ of only $28^{\circ}$ instead of the observed $75^{\circ}$

The most likely origin of the discrepancy between the observed and expected tilt angles is an overestimation of demagnetizing effects. The value of $\varphi_{t}=28^{\circ}$ is obtained assuming that the tilt is homogeneous over the stripe width. In reality, the demagnetizing effect is much smaller in the center than at the edges of the stripe, leading to a larger tilt angle in the center. We carried out micromagnetic simulations using the OOMMF code ${ }^{19}$ to obtain the magnetization profile of a 400-nm-wide, 5-nm-thick layer of $\mathrm{Ni}_{80} \mathrm{Fe}_{20}$ and for $\mathrm{NiFe}(5 \mathrm{~nm}) / \mathrm{Cu}(5 \mathrm{~nm}) / \mathrm{Co}(5 \mathrm{~nm})$ trilayers under an Oersted field of $7.4 \mathrm{mT}$. The results obtained for $\mathrm{NiFe}(\mathrm{Co})$ using an exchange constant $A$ of $1 \times 10^{-11} \mathrm{~J} / \mathrm{m}\left(3 \times 10^{-11} \mathrm{~J} / \mathrm{m}\right)$, a spontaneous magnetization $\mu_{0} M_{S}$ of $1 \mathrm{~T}(1.76 \mathrm{~T})$, and a vanishing magnetic anisotropy constant $K$ are shown in Fig. 2. The blue dash-dotted line (black solid line) shows the demagnetizing factor $\left(\varphi_{t}\right)$ for a single Py layer, as a function of transverse position. The average tilt angle is $26.6^{\circ}$, with a maximum of $32^{\circ}$ in the center of the stripe. As shown in previous studies, edge roughness can lead to a decrease in the transverse demagnetizing factor of several tens of percent. ${ }^{20}$ 


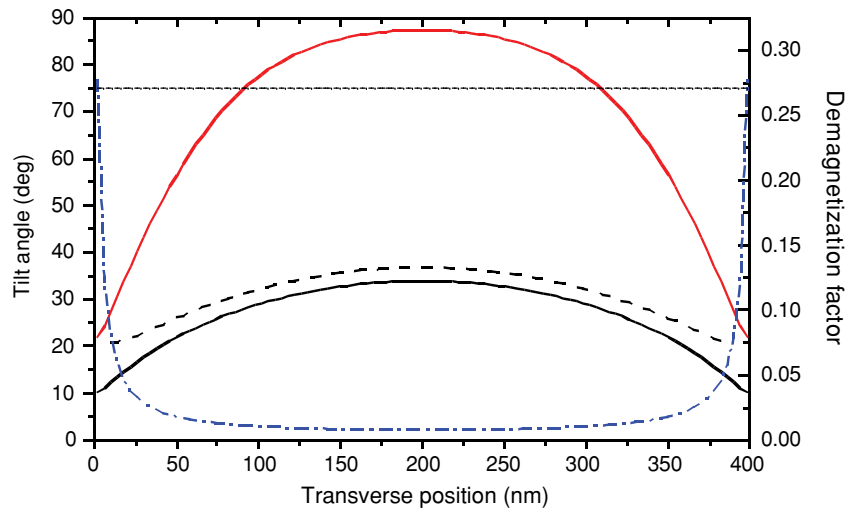

FIG. 2. (Color online) NiFe magnetization tilt angle as a function of the transverse position inside a 400-nm-wide stripe, obtained using the OOMMF code, for a transverse Oersted magnetic field of 7.4 mT. The experimental $\mathrm{NiFe}$ tilt angle is indicated with a dashed line. Different cases were considered: a single 5-nm-thick NiFe layer without edge roughness (black line), a 5-nm-think NiFe with a random edge roughness of 4-8 $\mathrm{nm}$ (dotted black line), and a $\mathrm{NiFe}(5 \mathrm{~nm}) / \mathrm{Cu}$ $(5 \mathrm{~nm}) / \mathrm{Co}(5 \mathrm{~nm})$ trilayer [red (light gray) line]. The demagnetization factor along the stripe is also shown [blue (dark gray) dash-dotted line, right $y$ axis].

The simulated magnetization profile obtained by adding a random lateral roughness of 4-8 nm (1-2 grid cells) at the stripe edges (dotted black line) leads only to a slight increase of the average tilt angle (to about $30^{\circ}$ ). Edge roughness is therefore not sufficient to explain the large experimental tilt. A better quantitative agreement with experiments can be obtained by taking into account the presence of the Co layer. Magnetostatic interactions between the $\mathrm{NiFe}$ and $\mathrm{Co}$ layers can significantly decrease the transverse demagnetizing effects with respect to single NiFe stripes. Part of the magnetic charges on the edges of the NiFe layer can be compensated by mirroring effects on the edges of the Co layer, as shown by micromagnetic simulations. ${ }^{21}$ Moreover, if the current is centered in the $\mathrm{Cu}$ layer, the Co magnetization tilt induced by $H_{\mathrm{Oe}}$ will be opposite to the one induced in the NiFe layer, further increasing the compensating effect of the Co magnetic charges. The average tilt angle obtained for the $\mathrm{NiFe}$ layer in the case of a $\mathrm{NiFe} / \mathrm{Cu} / \mathrm{Co}$ trilayer is around $69^{\circ}$, close to the experimental value, with a Co tilt angle (not shown) of about $-42^{\circ}$. The NiFe magnetization tilt strongly depends on the Co tilt angle. In the simulations in Fig. 2, the magnetic anisotropy in the Co layer was taken to be zero, which is justified by the polycrystalline nature of the Co, leading to the absence of an in-plane uniaxial anisotropy before patterning. A uniaxial Co anisotropy along the stripe axis of $50 \mathrm{~kJ} / \mathrm{m}^{3}$ would lead to a Co tilt angle of only $-9^{\circ}$ and a NiFe tilt of $42^{\circ}$.

At the onset of the current pulse, fast oscillations of the magnetization are observed in the time-resolved images [Fig. 1(i)]. These oscillations are due to the precession of the magnetization about the effective field. Figure 3 shows contrast-enhanced XMCD-PEEM images of the bottom section of the nanostripe in Fig. 1. Inhomogeneities in the dipolar interactions with the Co layer and in edge roughness lead to inhomogeneous magnetic contrast in the sections of the spinvalve nanostripe. Different parts of the nanostripe oscillate

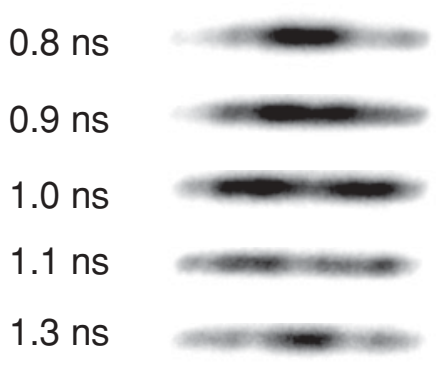

FIG. 3. Time-resolved XMCD-PEEM images of the lower $13-\mu \mathrm{m}$-long section of the nanostripe, taken at the indicated delays after the beginning of the positive part of the current pulse. Spatiotemporal variations of the XMCD contrast at a frequency of about $2 \mathrm{GHz}$ are visible. ${ }^{17}$

with different initial phases of the precessional motion. The exchange interaction between the different parts, however, leads to spatiotemporal variations of the magnetic contrast that resemble spin waves. The oscillatory and propagative nature of these contrast variations is more clearly visible in the accompanying movie. ${ }^{17}$ The excitation of spin waves by the Oersted field in spin-valve trilayers was predicted by Kim et al. ${ }^{22}$ and spin-wave-like features were observed using Lorentz microscopy on 30-nm-thick NiFe nanostripes upon current injection. ${ }^{23}$ Further micromagnetic simulations are necessary to understand these oscillations quantitatively, but our results show that time-resolved XMCD-PEEM is a very suitable technique to observe such magnetization oscillations.

In quasistatic measurements performed on similar nanostripes we have observed that current pulses with a density above $1.5-2 \times 10^{12} \mathrm{~A} / \mathrm{m}^{2}$ can induce nucleation of reversed domains in initially saturated nanostripe sections. ${ }^{12}$ Our present results suggest that the precession of the magnetization about $H_{\mathrm{Oe}}$ is possibly at the origin of this local magnetization reversal, similar to the magnetization reversal ${ }^{24}$ induced by transverse magnetic field pulses in magnetic nanostructures.

The magnetization tilt induced by the Oersted field and amplified by magnetostatic interactions should also have an influence on current-induced domain-wall motion in such trilayers. $^{10-12}$ The amplified Oersted field might stabilize transverse domain walls having their magnetization parallel to $H_{\mathrm{Oe}}$, like it was observed for field-induced domain-wall motion in trilayer nanostripes in the presence of a transverse magnetic field. ${ }^{25,26}$

In conclusion, we provide direct, time-resolved microscopic evidence of the effect of current-induced fields on the magnetic configuration of magnetic nanostripes. We show that the combination of Oersted fields and strong dipolar interactions that may exist in nanostripes comprised of several metallic layers produces and amplifies a large tilt of the magnetization. The quasistatic and precessional effects induced by the amplified Oersted field should be carefully considered when current pulses are applied to magnetic wires with different metallic layers, for instance, to study current-induced domain-wall motion. On the other hand, the effect of the Oersted field on magnetization reversal or magnetic domain-wall motion in future spintronic devices can 
be tailored by tuning the thickness of the different metallic layers.

We acknowledge the invaluable technical and experimental help of P. Perrier, D. Lepoittevin, L. Delrey, S. Pairis, T. Fournier, A. Hrabec, M. Bonfim, and W. Wernsdorfer. We thank A. Anane, J. Grollier, and R. Mattana for experimental help and useful discussions. We thank the European Synchrotron Radiation Facility (ESRF) and, in particular, the staff of beamline ID08, where several preliminary experiments were carried out. Nanofabrication was performed at the "Plateforme de Technologies Avancées" and at the Institut Néel/CNRS "Nanofab" facility, both in Grenoble. E.J. and J.C. acknowledge support by Spanish MICINN and Comunidad de Madrid through projects CSD 2007-00010 and S2009/MAT1726, respectively. V.U. was financially supported by Grant Nos. MSM0021630508, KAN400100701, and 2E13800101MSMT and by INGO Project No. LA287 of the Czech Ministry of Education. This work was partially supported by Grant No. ANR-07-NANO-034 "Dynawall."
${ }^{1}$ L. Berger, J. Appl. Phys. 55, 1954 (1984).

${ }^{2}$ J. Slonczewski, J. Magn. Magn. Mater. 159, L1 (1996).

${ }^{3}$ I. M. Miron, G. Gaudin, S. Auffret, B. Rodmacq, A. Schuhl, S. Pizzini, J. Vogel, and P. Gambardella, Nat. Mater. 9, 230 (2010).

${ }^{4}$ Y. Acremann, J. P. Strachan, V. Chembrolu, S. D. Andrews, T. Tyliszczak, J. A. Katine, M. J. Carey, B. M. Clemens, H. C. Siegmann, and J. Stöhr, Phys. Rev. Lett. 96, 217202 (2006).

${ }^{5}$ K. Ito, T. Devolder, C. Chappert, M. J. Carey, and J. A. Katine, J. Phys. D 40, 1261 (2007).

${ }^{6}$ D. Morecroft, I. A. Colin, F. J. Castaño, J. A. C. Bland, and C. A. Ross, Phys. Rev. B 76, 054449 (2007).

${ }^{7}$ P. J. Metaxas, A. Anane, V. Cros, J. Grollier, C. Deranlot, Y. Lemaître, S. Xavier, C. Ulysse, G. Faini, F. Petroff, and A. Fert, Appl. Phys. Lett. 97, 182506 (2010).

${ }^{8}$ A. Yamaguchi, T. Ono, S. Nasu, K. Miyake, K. Mibu, and T. Shinjo, Phys. Rev. Lett. 92, 077205 (2004).

${ }^{9}$ M. Kläui, P. O. Jubert, R. Allenspach, A. Bischof, J. A. C. Bland, G. Faini, U. Rüdiger, C. A. F. Vaz, L. Vila, and C. Vouille, Phys. Rev. Lett. 95, 026601 (2005).

${ }^{10}$ J. Grollier, P. Boulenc, V. Cros, A. Hamzic, A. Vaurès, A. Fert, and G. Faini, Appl. Phys. Lett. 83, 509 (2003).

${ }^{11}$ S. Pizzini, V. Uhlî̌, J. Vogel, N. Rougemaille, S. Laribi, V. Cros, E. Jiménez, J. Camarero, C. Tieg, E. Bonet, M. Bonfim, R. Mattana, C. Deranlot, F. Petroff, C. Ulysse, G. Faini, and A. Fert, Appl. Phys. Express 2, 023003 (2009).

${ }^{12}$ V. Uhlî́, S. Pizzini, N. Rougemaille, J. Novotný, V. Cros, E. Jiménez, G. Faini, L. Heyne, F. Sirotti, C. Tieg, A. Bendounan, F. Maccherozzi, R. Belkhou, J. Grollier, A. Anane, and J. Vogel, Phys. Rev. B 81, 224418 (2010).

${ }^{13}$ F. Sirotti, S. Girlando, P. Prieto, L. Floreano, G. Panaccione, and G. Rossi, Phys. Rev. B 61, R9221 (2000).
${ }^{14}$ M. Bonfim, G. Ghiringhelli, F. Montaigne, S. Pizzini, N. B. Brookes, F. Petroff, J. Vogel, J. Camarero, and A. Fontaine, Phys. Rev. Lett. 86, 3646 (2001).

${ }^{15}$ J. Vogel, W. Kuch, M. Bonfim, J. Camarero, Y. Pennec, F. Offi, K. Fukumoto, J. Kirschner, A. Fontaine, and S. Pizzini, Appl. Phys. Lett. 82, 2299 (2003).

${ }^{16}$ G. Schönhense, H. J. Elmers, S. A. Nepijko, and C. M. Schneider, Adv. Imaging Electron Phys. 142, 159 (2006).

${ }^{17}$ See supplemental material at [http://link.aps.org/supplemental/ 10.1103/PhysRevB.83.020406] for movies with the complete image series corresponding to Figs. 1 and 3.

${ }^{18}$ A. Aharoni, J. Appl. Phys. 83, 3432 (1998).

${ }^{19}$ M. J. Donahue and D. G. Porter, OOMMF user's guide, version 1.0, Interagency Report No. NISTIR 6376, NIST, Gaithersburg, MD, 1999.

${ }^{20}$ R. D. Cowburn, D. K. Koltsov, A. O. Adeyeye, and M. E. Welland, J. Appl. Phys. 87, 7067 (2000).

${ }^{21}$ J. M. B. Ndjaka, A. Thiaville, and J. Miltat, J. Appl. Phys. 105, 023905 (2009).

${ }^{22}$ W. J. Kim, S. M. Seo, T. D. Lee, and K. J. Lee, J. Magn. Magn. Mater. 310, 2032 (2007).

${ }^{23}$ Y. Togawa, T. I. Kimura, K. Harada, T. Matsuda, A. Tonomura, Y. Otani, and T. Akashi, Appl. Phys. Lett. 92, 012505 (2008).

${ }^{24}$ H. W. Schumacher, C. Chappert, P. Crozat, R. C. Sousa, P. P. Freitas, J. Miltat, J. Fassbender, and B. Hillebrands, Phys. Rev. Lett. 90, 017201 (2003).

${ }^{25}$ M. T. Bryan, T. Schrefl, D. Atkinson, and D. A. Allwood, J. Appl. Phys. 103, 073906 (2008).

${ }^{26}$ S. Glathe, I. Berkov, T. Mikolajick, and R. Mattheis, Appl. Phys. Lett. 93, 162505 (2008). 